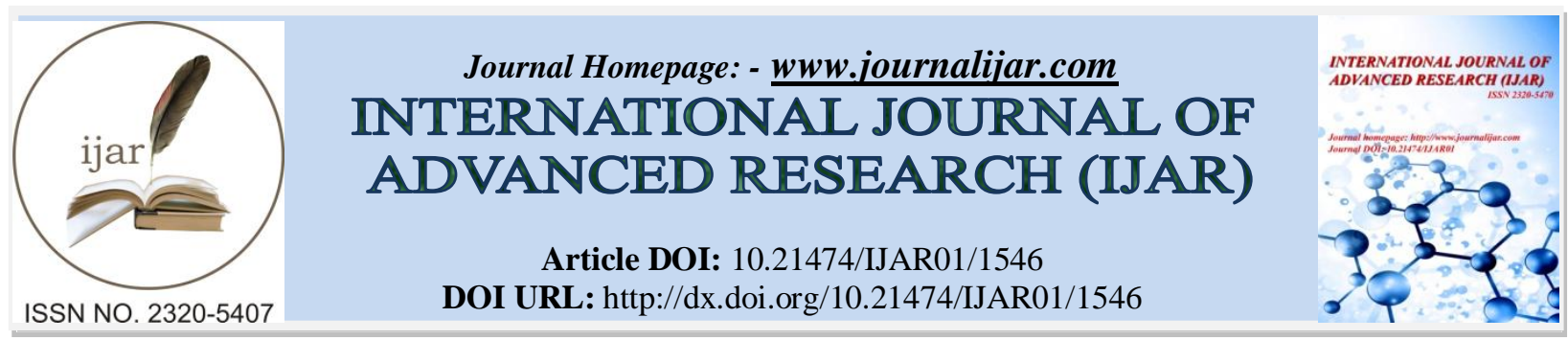

RESEARCH ARTICLE

\title{
SOIL EVALUATIOIN FOR AGRICULTURE DEVELOPMENT IN AN AREA OF NORTHWESTERN COAST, EGYPT.
}

Riad, M. H.

Soils, Water and Environment Res. Inst., Agric.Res.Center, Giza, Egypt.

\section{Manuscript Info}

Manuscript History

Received: 16 July 2016

Final Accepted: 23 August 2016

Published: September 2016

Key words:-

Land Evaluation, Agriculture

development, Northwestern coast of

Egypt, RS, GIS, Geomorphologic units.

\section{Abstract}

The aim of the present work is to evaluate some land resources in an area of northwestern coast, Egypt for agriculture development. The study area is extend from El-Alamin in the east to El-Dabaa on the west and lies between longitudes $28^{\circ} 24^{\prime} 00^{\prime \prime}$ and $29^{\circ} 00^{\prime} 30^{\prime \prime} \mathrm{E}$ and latitudes $30^{\circ} 44^{\prime} 00^{\prime \prime}$ and $31^{\circ} 01^{\prime} 30^{\prime \prime} \mathrm{N}$, with a total area of $719.4 \mathrm{Km}^{2}$ (71940 hectares).

RS and GIS techniques were used for identifying and interpretation the geomorphologic units of the study area. The geomorphologic map of the area was produced using digital image processing of Landsat $\mathrm{ETM}^{+}$, TIN, DEM, geological map and field observation data. Accordingly, three main geomorphologic units are recognized: gently sloping marine plain, lacustrine plain and terraced marine plain.

Thirty four soil profiles (representing the geomorphologic units) were selected and allocated by the portable Global Positioning System (GPS) and the soils are morphologically described in the field. 63 soil samples have been collected from the profiles according to the morphological variations. The physical and chemical properties of soil samples were determined in the lab.

The current suitability of the studied soils was estimated by matching between the present land characteristics and their ratings outlined by Sys and Verhey (1978) and Sys et al (1991).

Suitability indices and classification of the studied soils revealed that there are two suitability classes, i.e. marginally suitable (S3) and currently not suitable (N1). The soil limiting factors in the study area are depth, texture, topography, $\mathrm{CaCO}_{3}$ and salinity \& alkalinity with slight to severe and very severe intensity for soil limitations.

Further land improvements are required to correct or reduce the severity of limitations exiting in the studied area, such as: a) leaching of soils salinity and reclamation of soil sodicity, b) continuous application of organic, manure to improve soil physico-chemical properties and fertility status, and c) application of modern irrigation system, i.e. drip and sprinkler. By applying the previous improvement practices, potential suitability of the studied soils could raised to moderately suitable (S2) and marginally suitable (S3). 


\section{Introduction:-}

The continuous increase of human pressure on our limited natural resources, including water and cultivated area requires proper management of such resources. The agricultural expansion outside the old Nile valley is one of the main objects of the Egyptian national plan. A great attention is directed to the northwestern coastal area (NWCE) of Egypt, due to it's diverse characteristics. Therefore, studies on management of natural resources in such regions are considered of vital importance (Ali, 2008). Generally, sustainable development is a primary objective and an urgent problem to be addressed by our society (Campanga, 2005).

According to FAO methodology (1976); land suitability is strongly related to land qualities including erosion resistance, water availability and flood hazards which are derived from slope and length, rainfall and soil texture. Sys et al. (1991) suggested a parametric evaluation system for irrigation which was primarily based on physical and chemical soil properties.

The main objective of this research is to produce the geomorphological map as well as to evaluate land suitability for irrigated agriculture in an area of the northwestern coastal zone, Egypt using remote sensing technique.

\section{Study area:-}

The study area is located in the northwestern coast of Egypt. It extend from El- Alamin in the east to El- Dabaa on the west and lies between longitudes $28^{\circ} 24^{\prime} 00^{\prime \prime}$ and $29^{\circ} 00^{\prime} 30^{\prime \prime} \mathrm{E}$ and latitudes $30^{\circ} 44^{\prime} 00^{\prime \prime}$ and $31^{\circ} 01^{\prime} 30^{\prime \prime} \mathrm{N}$, with a total area of $719.4 \mathrm{Km}^{2}$ (71940 hectares); Fig. (1).

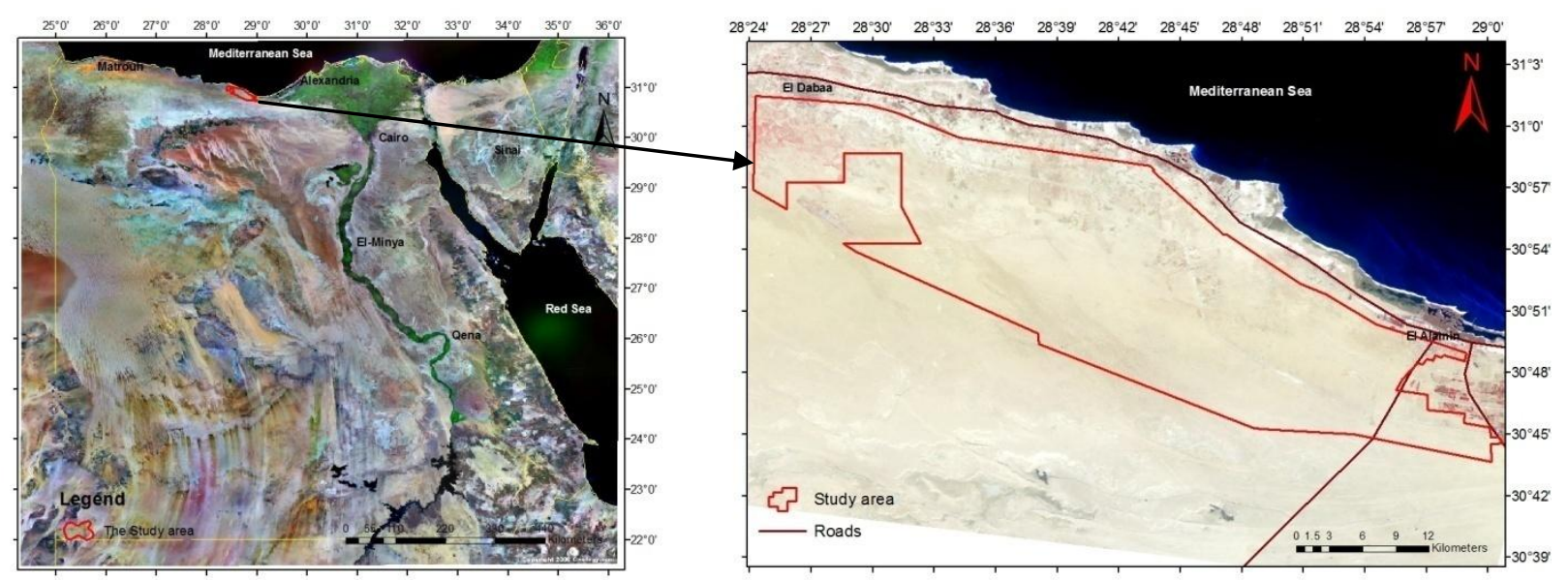

\section{Climate:-}

Fig. 1:- Location map of the study area.

The climatic conditions of the study area are typically arid to semi-arid, characterized by a long hot dry summer, mild winter with little rainfall, high evaporation with moderately to high relative humidity. Table (1) postulates the average meteorological data (1971-2000) from Alexandria station (data from ministry of agriculture and land reclamation, 2007). The annual rate maximum temperature is $25^{\circ} \mathrm{C}$. The Mediterranean coastal zone of Egypt receives noticeable amounts of rainfall, especially in winter. The annual rainfall is low as it does not exceed 16. 6 $\mathrm{mm}$. The maximum monthly rainfall is $55.6 \mathrm{~mm}$ in December. Precipitation is considered as the main source of recharge of groundwater aquifers in the northwestern Mediterranean coastal zone and affects greatly the amount of water stored in such aquifers.

The relative humidity plays an important role in the amount of evaporation and evapotranspiration. The values of relative humidity are relatively high in summer months. Prevailing winds at the study area are chiefly directed southwest in the winter months while being northwest in the summer months. The lowest and highest wind velocities are recorded in October and March, respectively. Evaporation data indicate that the lowest values of evaporation are recorded in January while the highest values are monitored in July.With such high annual evaporation, both irrigation water and energy costs required for irrigation would be very high. 
Table 1:- Mean monthly climatological data of Alexandria station (1971-2000).

\begin{tabular}{|c|c|c|c|c|c|c|c|}
\hline \multirow[t]{2}{*}{ Month } & \multicolumn{3}{|c|}{ Temperature $\left(\mathbf{C}^{\circ}\right)$} & \multirow{2}{*}{$\begin{array}{c}\text { Rainfall } \\
(\mathbf{m m})\end{array}$} & \multirow{2}{*}{$\begin{array}{c}\text { Relative } \\
\text { Humidity } \\
(\%)\end{array}$} & \multirow{2}{*}{$\begin{array}{c}\text { Wind } \\
\text { Velocity } \\
(\mathbf{k m} / \mathbf{h})\end{array}$} & \multirow{2}{*}{$\begin{array}{c}\text { Avg. Eto } \\
(\mathbf{m m} / \mathbf{d})\end{array}$} \\
\hline & Max. & Min. & Mean. & & & & \\
\hline Jan. & 18,40 & 9,10 & 13,50 & 54,90 & 70,00 & 7,50 & 2,20 \\
\hline Feb. & 19,30 & 9,30 & 14,10 & 26,60 & 68,00 & 7,50 & 2,60 \\
\hline Mar. & 21,30 & 10,80 & 15,80 & 12,90 & 65,00 & 7,80 & 3,40 \\
\hline Apr. & 23,50 & 13,10 & 18,30 & 4,20 & 65,00 & 7,30 & 4,10 \\
\hline May. & 26,60 & 16,40 & 21,20 & 1,50 & 67,00 & 6,80 & 4,90 \\
\hline Jun. & 28,60 & 20,20 & 24,30 & 0,00 & 69,00 & 6,80 & 5,70 \\
\hline Jul. & 29,70 & 22,00 & 25,90 & 0,00 & 72,00 & 7,40 & 5,80 \\
\hline Aug. & 30,60 & 22,70 & 26,50 & 0,30 & 71,00 & 6,80 & 5,50 \\
\hline Sep. & 29,60 & 21,10 & 25,60 & 1,00 & 68,00 & 6,20 & 4,90 \\
\hline Oct. & 27,60 & 17,60 & 22,50 & 9,30 & 68,00 & 5,30 & 3,70 \\
\hline Nov. & 24,20 & 14,40 & 19,10 & 33,10 & 69,00 & 5,80 & 2,70 \\
\hline Dec. & 20,30 & 10,80 & 15,20 & 55,60 & 70,00 & 7,00 & 2,30 \\
\hline mean & 25.0 & 15.6 & 20.16 & 16.6 & 62.83 & 6.85 & 4.07 \\
\hline
\end{tabular}

ETo: Reference Evapotranspiration

\section{Geology:-}

The northern part of Egypt including, the north Western Desert, the Nile Delta and north Sinai lie in the unstable shelf area. The main part of Egypt west of the river Nile is covered by thick sequences of relatively undisturbed sedimentary strata of Paleozoic, Mesozoic and Cenozoic age (Said, 1990). The north part of the Western Desert is covered mainly by thin blanket of Miocene rocks forming a vast persistent limestone plateau. It extends from the western side of the Nile valley and delta in the east to El-Salum in the west and the Mediterranean coastal plain in the north to the Qattara and Siwa depression in the south (El-Bastwasy, 2008). The geology surface of the study area is essentially dominated by sedimentary rocks of Tertiary and Quaternary ages (Fig. 2). The Quaternary is exposed in the coastal plain, wadis and raised beaches. The Pliocene and Miocene of the Tertiary is exposed major part of the tableland, the Miocene is forming the surface beds of the tableland. The geology is characterized by the presence of a plateau formed, essentially of Tertiary Miocene, mainly composed of limestone and sand stone. The coastal zone to the north of the Miocene plateau is covered by Pliocene deposits that consists of calcareous deposits overlie limestone. Quaternary deposits are rest with conformable and / or unconformable relation of the Tertiary deposits. These deposits are mainly represented by the Holocene deposits of coastal sand dunes, lagoonal and alluvial deposits and the Pleistocene oolitic limestone ridges and old lagoonal deposits (Zahran, 2008).

\section{Materials and methods:-}

The following data were used for the study:-

1. Remotely sensed data: Landsat-8 ETM multispectral image dated (Jan, 2015) with resolution of $30 \mathrm{~m}$ (Fig.1).

2. This image is orthorectified Landsat imagery provided by the Global Land Cover Facility (GLCF; http://glcf.umiacs.umd.edu/).

3. Topographic map $(1: 50,000)$ (EGACS, 1989).

4. Geological map of the study area (Fig. 2).

5. Digital elevation model (DEM) that covered the area of interest (Fig.3) has been cut and separated from ASTER GDEM (http://www.gdem.aster.ersdac.or.jp, Access date: Oct. 2010).

\section{Field work:-}

Field investigations were carried out in the study area using geological map with 1:500.000 scales (CONCO, 1987), topographic map with 1:50,000 scales produced by the Mineral Resources Authority of Egypt (1994) and Satellite data (landsat $\mathrm{ETM}^{+}$image with path and row 179 and 38, respectively). Different geomorphologic units were identified in the study area. 


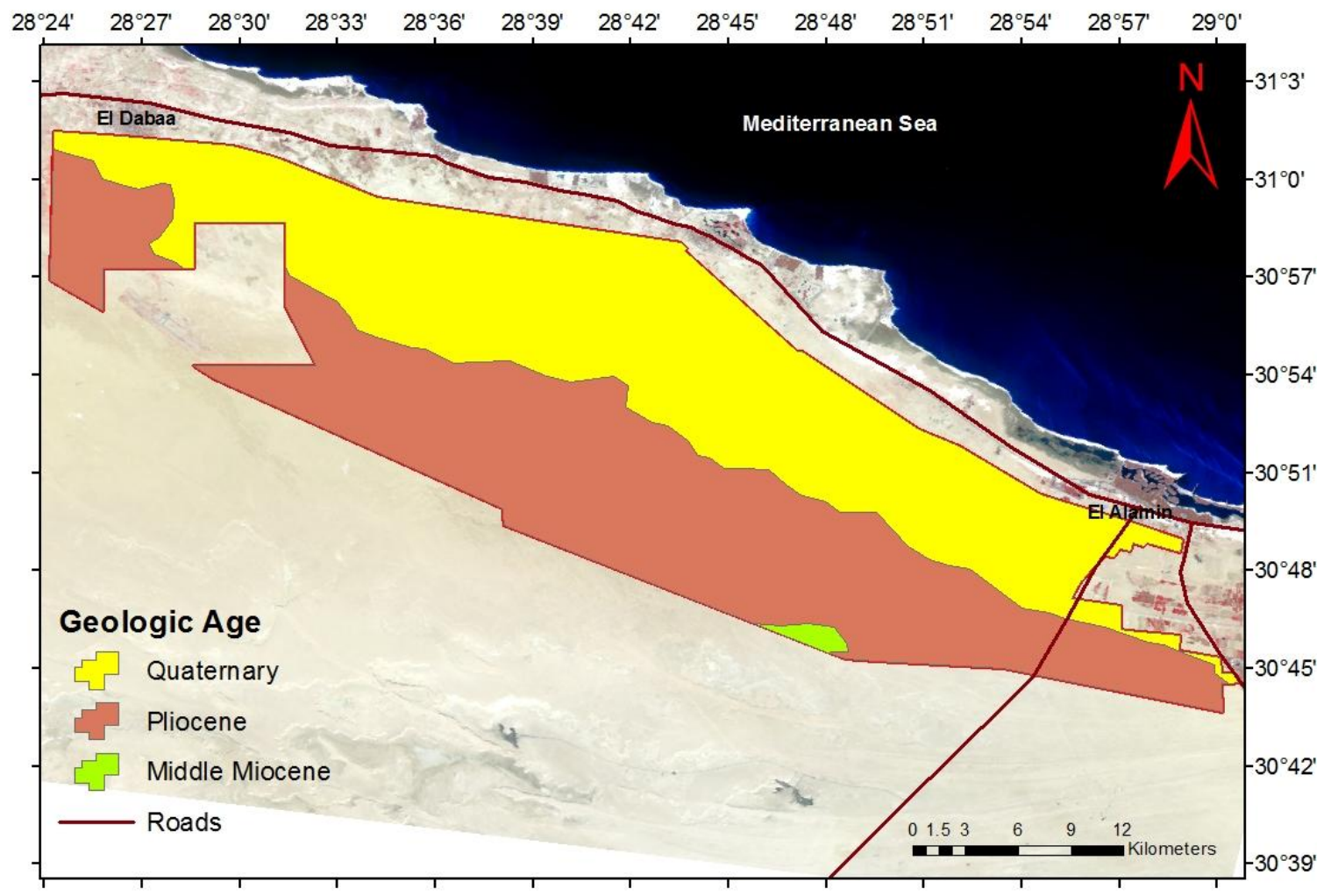

Fig. 2:- Geological map of the study area, (modified after CONCO, 1987)

Thirty four soil profiles were selected representing the identified geomorphological units, and allocated by the Global Positioning System (GPS). Detailed morphological descriptions of the soil profiles were recorded on the base of FAO (1990). Representative 63 soil samples have been collected from the studied soil profiles according to the morphological variations.

\section{Laboratory analyses:-}

Soil samples were air dried, crushed and sieved through a $2 \mathrm{~mm}$ sieve. The fine earth fractions $(<2 \mathrm{~mm})$ were used for physical and chemical analyses.

Particle size distribution was determined using the international pipette method (USDA, 2004). Calcium carbonate content was measured using the Collin's Calcimeter method (USDA, 2004). Gypsum was determined by the acetone method (Richards, 1954).

Soil $\mathrm{pH}$ was determined in the soil paste using $\mathrm{pH}$ meter and total salinity was expressed as electrical conductivity (ECe dS/m) according to (USDA, 2004).

\section{Land evaluation:-}

The land capability and suitability evaluation was assessed based on Sys and Verheye (1978) and Sys et al (1991). 


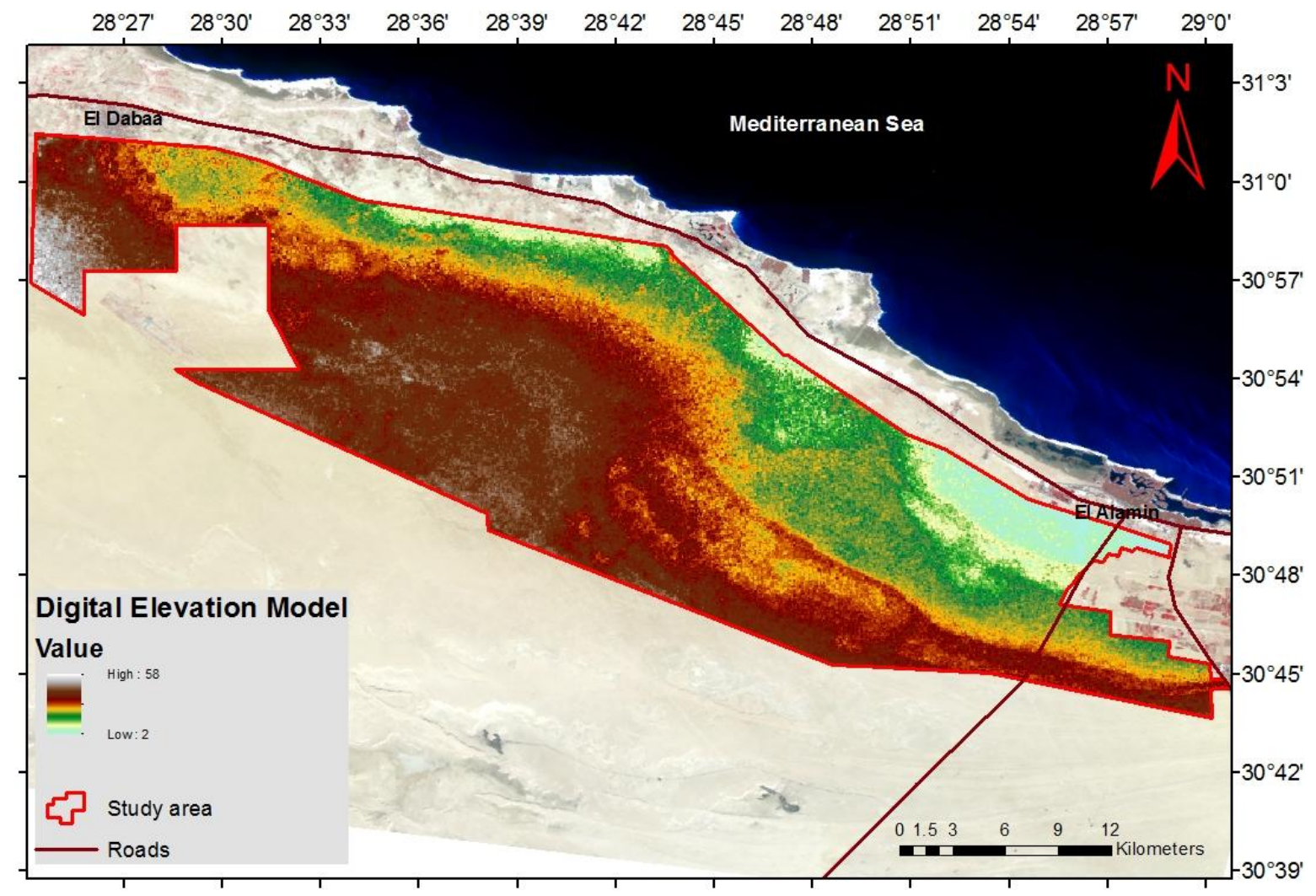

\section{Results and Discussions:- \\ Geomorphologic Setting:-}

Fig. 3:- Digital elevation model of the study area.

The Northwest Coast of Egypt forms a belt about $20 \mathrm{Km}$ deep, which extends for about $500 \mathrm{~km}$ between Amria (20 $\mathrm{Km}$ west of El-Alexandria) and El-Salloum near the Libyan borders. The landscape is distinguished into a northern coastal plain and a southern tableland. The coastal plain stretches in general east-west direction, bounded by the sea to the north and tableland in the south. The land surface of the frontal plain is slope in a northward direction. However, the slope of the land surface in the study area is mostly range from 1 to $5 \%$ which is considered as gentle slope. This gentle slope does not accelerate surface runoff where the area has an indistinct drainage pattern. The surface runoff is captured by low lying depression where most of this surface storage evaporates (Fehlberg and Stahr, 1985).

The geomorphlogical units of the study area are shown in Fig. (4) and described as follows:

\section{Gently sloping marine plain unit:}

It represents low and medium relief marine deposits with gently sloping surface and located in the northern part of the study area. However, the subsoil layers are formed locally from marine limestone (El-Bastwasy, 2008).

\section{Lacustrine pain unit:-}

The lacustrine deposits are formed when still water in lakes permits fine particles (fine sand, silt, and clay) to settle out and to form lacustrine plain. These deposits get exposed by elevation of old lakebeds. Lacustrine deposits are very well sorted, devoid of coarse particles such as coarse sand or gravels, and are characterized by thin layers that reflect annual deposition of sediments.

\section{Terraced marine plain unit:}

It characterized by relatively high elevation and located between marine plain unit and the limestone plateaus area. Terraces are result from marine erosion along rocky coast lines and the main factor for the formation of marine terraces, derived sea level fluctuations can indicate former climate changes. 


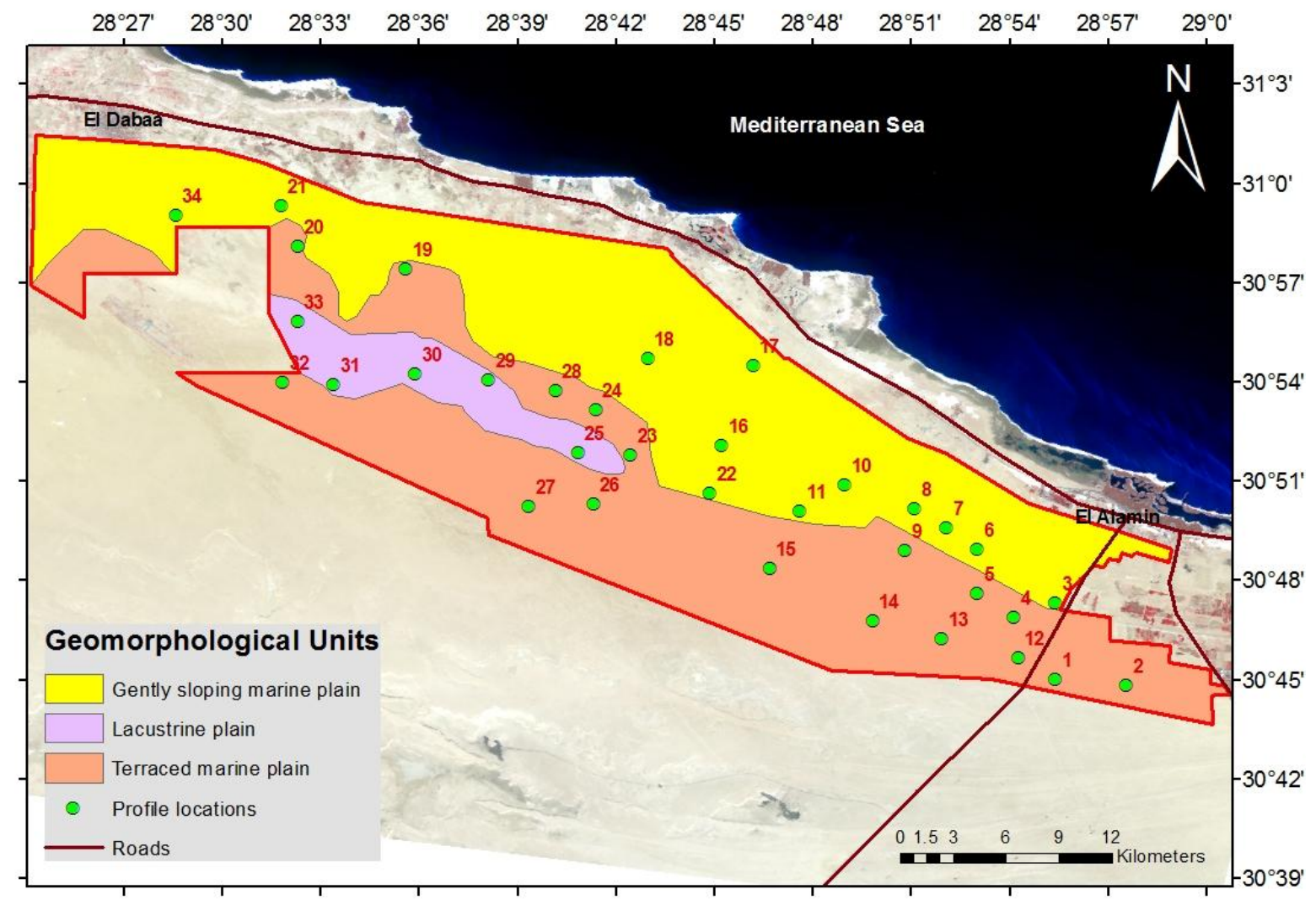

Fig. 4:- Geomorphological units and soil profile locations of the study area.

\section{Soil characteristics:-}

* The soil characteristics of the study area are the basis for land use evaluation. These characteristics can be summarized as follows:

* The study shows that geomorphological unit type is the main factor governing the soil morphology and chemistry. A brief morphological description and some physical and chemical characteristics of the study soil profiles are illustrated in Tables (2 and 3).

* The soil profiles are mostly deep in marine and lacustrine plain units, while in terraced unit is shallow depth.

* The gravel contents are relatively high in terraced unit followed by gently sloping marine plain unit, while lacustrine unit has low gravel contents. Soil textures of the different geomorphological units are range from sand to sandy clay loam (Table 3).

* The soil chemical analyses can be summarized as follows (Table 3):-

* The average soil reaction $(\mathrm{pH})$ values are fluctuated between neutral and moderately alkaline.

* The soil salinity ranges from non saline to very strongly saline in marine and lacustrine plain units. However, the terraced unit is mostly non- and moderately saline.

* The soil profiles are mostly having high $\mathrm{CaCO}_{3}$ in all geomorphic units (ranging from 16.8 to $70.2 \%$ ), while gypsum content is low and moderate in terraced unit and high in some profiles in marine and lacustrine plain units (ranging from 0.16 to $11.41 \%$ ). 
Table 2:- Morphological description of soil profiles in the study area.

\begin{tabular}{|c|c|c|c|c|c|c|c|c|c|c|c|c|}
\hline \multirow{3}{*}{ 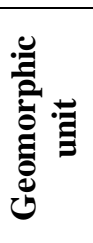 } & \multirow{3}{*}{$\dot{0}$} & \multirow{3}{*}{$\begin{array}{l}\text { Depth } \\
\text { (cm) }\end{array}$} & \multirow{3}{*}{$\begin{array}{l}\text { Soil } \\
\text { Color } \\
\text { (dry) }\end{array}$} & \multirow{3}{*}{ 莺 } & \multicolumn{4}{|c|}{ Consistence } & \multirow{2}{*}{\multicolumn{2}{|c|}{$\begin{array}{l}\text { Secondary } \\
\text { formation }\end{array}$}} & \multirow{3}{*}{ 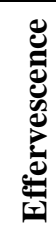 } & \multirow{3}{*}{$\underset{0}{\stackrel{B}{E}}$} \\
\hline & & & & & \multirow[b]{2}{*}{ Dry } & \multirow[b]{2}{*}{ Moist } & \multicolumn{2}{|c|}{ Wet } & & & & \\
\hline & & & & & & & ST & PL & $\mathbf{K}$ & $\mathbf{Y}$ & & \\
\hline \multirow{12}{*}{ 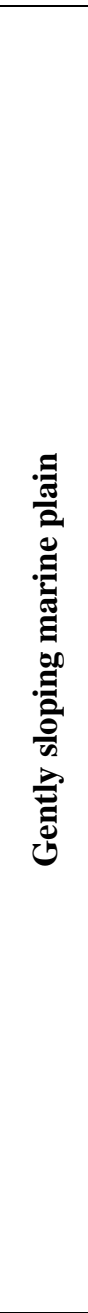 } & 3 & $\begin{array}{c}0-25 \\
25-70\end{array}$ & $\begin{array}{l}\text { 10YR7/3 } \\
\text { 10YR6/3 }\end{array}$ & $\begin{array}{l}\text { MA } \\
\text { MA }\end{array}$ & $\begin{array}{l}\text { SO } \\
\text { SO }\end{array}$ & $\begin{array}{l}\text { FR } \\
\text { FR }\end{array}$ & $\begin{array}{l}\text { NST } \\
\text { NST }\end{array}$ & $\begin{array}{l}\text { NPL } \\
\text { NPL }\end{array}$ & $\begin{array}{l}\mathrm{M} \\
\mathrm{C}\end{array}$ & $\begin{array}{l}- \\
-\end{array}$ & $\begin{array}{l}\mathrm{St} \\
\mathrm{St}\end{array}$ & $\begin{array}{c}\mathrm{CW} \\
-\end{array}$ \\
\hline & 6 & $\begin{array}{c}0-40 \\
40-80 \\
80-105 \\
105-150\end{array}$ & $\begin{array}{l}10 \mathrm{YR} 7 / 3 \\
10 \mathrm{YR} 6 / 4 \\
10 \mathrm{YR} 7 / 3 \\
7.5 \mathrm{YR} 7 / 3\end{array}$ & $\begin{array}{l}\text { MA } \\
\text { MA } \\
\text { MA } \\
\text { MA }\end{array}$ & $\begin{array}{c}\text { SHA } \\
\text { HA } \\
\text { HA } \\
\text { HA }\end{array}$ & $\begin{array}{c}\mathrm{FR} \\
\mathrm{Fi} \\
\mathrm{Fi} \\
\mathrm{Fi}\end{array}$ & $\begin{array}{l}\text { ST } \\
\text { ST } \\
\text { ST } \\
\text { ST }\end{array}$ & $\begin{array}{l}\text { PL } \\
\text { PL } \\
\text { PL } \\
\text { PL }\end{array}$ & $\begin{array}{l}\mathrm{M} \\
\mathrm{M} \\
\mathrm{M} \\
\mathrm{M}\end{array}$ & $\begin{array}{l}\mathrm{F} \\
\mathrm{C} \\
\mathrm{F} \\
\mathrm{C}\end{array}$ & $\begin{array}{l}\text { St } \\
\text { St } \\
\text { St } \\
\text { St }\end{array}$ & $\begin{array}{c}\text { CW } \\
\text { AS } \\
\text { AS } \\
-\end{array}$ \\
\hline & 7 & $\begin{array}{c}0-30 \\
30-100\end{array}$ & $\begin{array}{l}\text { 10YR5/4 } \\
10 Y R 7 / 3\end{array}$ & $\begin{array}{l}\text { MA } \\
\text { MA }\end{array}$ & $\begin{array}{l}\text { SO } \\
\text { SO }\end{array}$ & $\begin{array}{l}\text { FR } \\
\text { FR }\end{array}$ & $\begin{array}{l}\text { SST } \\
\text { SST }\end{array}$ & $\begin{array}{l}\text { SPL } \\
\text { SPL }\end{array}$ & $\begin{array}{l}\mathrm{M} \\
\mathrm{M}\end{array}$ & $\begin{array}{l}\mathrm{F} \\
\mathrm{C}\end{array}$ & $\begin{array}{l}\mathrm{St} \\
\mathrm{St}\end{array}$ & $\begin{array}{c}\text { AS } \\
-\end{array}$ \\
\hline & 8 & $\begin{array}{c}0-50 \\
50-70\end{array}$ & $\begin{array}{l}10 \mathrm{YR} 7 / 3 \\
10 \mathrm{YR} 7 / 3\end{array}$ & $\begin{array}{l}\text { MA } \\
\text { MA }\end{array}$ & $\begin{array}{l}\mathrm{SO} \\
\mathrm{SO}\end{array}$ & $\begin{array}{l}\text { FR } \\
\text { FR }\end{array}$ & $\begin{array}{l}\text { SST } \\
\text { SST }\end{array}$ & $\begin{array}{l}\text { SPL } \\
\text { NPL }\end{array}$ & $\begin{array}{l}\mathrm{M} \\
\mathrm{M}\end{array}$ & - & $\begin{array}{l}\mathrm{St} \\
\mathrm{St}\end{array}$ & $\begin{array}{c}\mathrm{CW} \\
-\end{array}$ \\
\hline & 10 & $\begin{array}{c}0-30 \\
30-80 \\
80-120\end{array}$ & $\begin{array}{l}10 \mathrm{YR} 7 / 3 \\
10 \mathrm{YR} 7 / 3 \\
10 \mathrm{YR} 7 / 3\end{array}$ & $\begin{array}{l}\text { MA } \\
\text { MA } \\
\text { MA }\end{array}$ & $\begin{array}{l}\text { SO } \\
\text { SO } \\
\text { SO }\end{array}$ & $\begin{array}{l}\text { FR } \\
\text { FR } \\
\text { FR }\end{array}$ & $\begin{array}{l}\text { SST } \\
\text { NST } \\
\text { SST }\end{array}$ & $\begin{array}{l}\text { SPL } \\
\text { NPL } \\
\text { SPL }\end{array}$ & $\begin{array}{l}\mathrm{M} \\
\mathrm{M} \\
\mathrm{M}\end{array}$ & $\begin{array}{l}- \\
- \\
-\end{array}$ & $\begin{array}{l}\mathrm{St} \\
\mathrm{St} \\
\mathrm{St}\end{array}$ & $\begin{array}{c}\mathrm{CW} \\
\mathrm{CW} \\
-\end{array}$ \\
\hline & 11 & $\begin{array}{c}0-30 \\
30-100\end{array}$ & $\begin{array}{l}10 \mathrm{YR} 7 / 3 \\
10 \mathrm{YR} 7 / 3\end{array}$ & $\begin{array}{l}\text { MA } \\
\text { MA }\end{array}$ & $\begin{array}{l}\text { SO } \\
\text { SO }\end{array}$ & $\begin{array}{l}\text { FR } \\
\text { FR }\end{array}$ & $\begin{array}{l}\text { NST } \\
\text { NST }\end{array}$ & $\begin{array}{l}\text { NPL } \\
\text { NPL }\end{array}$ & $\begin{array}{l}\mathrm{M} \\
\mathrm{C}\end{array}$ & - & $\begin{array}{l}\mathrm{St} \\
\mathrm{St}\end{array}$ & $\begin{array}{c}\mathrm{CW} \\
-\end{array}$ \\
\hline & 16 & $\begin{array}{c}0-25 \\
25-40 \\
40-60 \\
60-85 \\
85-120\end{array}$ & $\begin{array}{l}10 \mathrm{YR} 5 / 8 \\
10 \mathrm{YR} 7 / 3 \\
10 \mathrm{YR} 7 / 3 \\
10 \mathrm{YR} 7 / 3 \\
10 \mathrm{YR} 7 / 3\end{array}$ & $\begin{array}{l}\text { MA } \\
\text { MA } \\
\text { MA } \\
\text { MA } \\
\text { MA }\end{array}$ & $\begin{array}{c}\text { SO } \\
\text { HA } \\
\text { SHA } \\
\text { SO } \\
\text { SO }\end{array}$ & $\begin{array}{c}\text { FR } \\
\text { Fi } \\
\text { Fi } \\
\text { FR } \\
\text { FR }\end{array}$ & $\begin{array}{c}\text { SST } \\
\text { ST } \\
\text { ST } \\
\text { SST } \\
\text { SST }\end{array}$ & $\begin{array}{l}\text { SPL } \\
\text { PL } \\
\text { PL } \\
\text { SPL } \\
\text { SPL }\end{array}$ & $\begin{array}{l}\mathrm{C} \\
\mathrm{M} \\
\mathrm{M} \\
\mathrm{M} \\
\mathrm{M}\end{array}$ & $\begin{array}{l}- \\
- \\
- \\
- \\
-\end{array}$ & $\begin{array}{l}\text { St } \\
\text { St } \\
\text { St } \\
\text { St } \\
\text { St }\end{array}$ & $\begin{array}{c}\text { AS } \\
\text { CW } \\
\text { CW } \\
\text { CW } \\
-\end{array}$ \\
\hline & 17 & $\begin{array}{c}0-30 \\
30-70 \\
70-120\end{array}$ & $\begin{array}{l}10 \mathrm{YR} 7 / 3 \\
10 \mathrm{YR} 5 / 8 \\
10 \mathrm{YR} 7 / 3\end{array}$ & $\begin{array}{l}\text { MA } \\
\text { MA } \\
\text { MA }\end{array}$ & $\begin{array}{l}\text { SO } \\
\text { SO } \\
\text { LO }\end{array}$ & $\begin{array}{c}\text { SHA } \\
\text { SHA } \\
\text { LO }\end{array}$ & $\begin{array}{l}\text { SST } \\
\text { SST } \\
\text { SST }\end{array}$ & $\begin{array}{l}\text { SPL } \\
\text { SPL } \\
\text { SPL }\end{array}$ & $\begin{array}{l}\mathrm{M} \\
\mathrm{M} \\
\mathrm{M}\end{array}$ & $\begin{array}{l}- \\
- \\
-\end{array}$ & $\begin{array}{l}\text { St } \\
\text { St } \\
\text { St }\end{array}$ & $\begin{array}{c}\mathrm{CS} \\
\mathrm{CS} \\
-\end{array}$ \\
\hline & 18 & $\begin{array}{c}0-25 \\
25-70\end{array}$ & $\begin{array}{l}\text { 10YR7/3 } \\
\text { 10YR5/8 }\end{array}$ & $\begin{array}{l}\text { MA } \\
\text { MA }\end{array}$ & $\begin{array}{l}\text { LO } \\
\text { LO }\end{array}$ & $\begin{array}{l}\text { LO } \\
\text { LO }\end{array}$ & $\begin{array}{l}\text { NST } \\
\text { NST }\end{array}$ & $\begin{array}{l}\text { NPL } \\
\text { NPL }\end{array}$ & $\begin{array}{l}M \\
M\end{array}$ & $\begin{array}{l}- \\
-\end{array}$ & $\begin{array}{l}\mathrm{St} \\
\mathrm{St}\end{array}$ & $\begin{array}{c}\mathrm{CW} \\
-\end{array}$ \\
\hline & 21 & $\begin{array}{c}0-30 \\
30-100\end{array}$ & $\begin{array}{l}10 \text { YR5/4 } \\
10 \text { YR7/3 }\end{array}$ & $\begin{array}{l}\text { MA } \\
\text { MA }\end{array}$ & $\begin{array}{c}\text { SHA } \\
\text { SO }\end{array}$ & $\begin{array}{l}\mathrm{Fi} \\
\mathrm{FR}\end{array}$ & $\begin{array}{c}\text { ST } \\
\text { SST }\end{array}$ & $\begin{array}{l}\text { PL } \\
\text { SPL }\end{array}$ & $\begin{array}{l}\mathrm{M} \\
\mathrm{M}\end{array}$ & $\begin{array}{l}\mathrm{F} \\
\mathrm{C}\end{array}$ & $\begin{array}{l}\mathrm{St} \\
\mathrm{St}\end{array}$ & $\begin{array}{c}\mathrm{CW} \\
-\end{array}$ \\
\hline & 22 & $\begin{array}{c}0-30 \\
30-70 \\
70-120\end{array}$ & $\begin{array}{l}10 \mathrm{YR} 7 / 3 \\
10 \mathrm{YR} 7 / 3 \\
10 \mathrm{YR} 7 / 3\end{array}$ & $\begin{array}{l}\text { MA } \\
\text { MA } \\
\text { MA }\end{array}$ & $\begin{array}{l}\text { SO } \\
\text { SO } \\
\text { SO }\end{array}$ & $\begin{array}{l}\text { FR } \\
\text { FR } \\
\text { FR }\end{array}$ & $\begin{array}{l}\text { NST } \\
\text { SST } \\
\text { SST }\end{array}$ & $\begin{array}{l}\text { NPL } \\
\text { SPL } \\
\text { SPL }\end{array}$ & $\begin{array}{l}\mathrm{C} \\
\mathrm{M} \\
\mathrm{M}\end{array}$ & $\begin{array}{l}\mathrm{F} \\
\mathrm{C} \\
\mathrm{F}\end{array}$ & $\begin{array}{l}\text { St } \\
\text { St } \\
\text { St }\end{array}$ & $\begin{array}{c}\mathrm{CW} \\
\mathrm{CW} \\
-\end{array}$ \\
\hline & 34 & $\begin{array}{c}0-40 \\
40-110\end{array}$ & $\begin{array}{l}\text { 10YR7/3 } \\
10 Y R 7 / 3\end{array}$ & $\begin{array}{l}\text { MA } \\
\text { MA }\end{array}$ & $\begin{array}{l}\text { SHA } \\
\text { SHA }\end{array}$ & $\begin{array}{l}\mathrm{Fi} \\
\mathrm{Fi}\end{array}$ & $\begin{array}{l}\text { ST } \\
\text { ST }\end{array}$ & $\begin{array}{l}\text { PL } \\
\text { PL }\end{array}$ & $\begin{array}{l}\mathrm{C} \\
\mathrm{M}\end{array}$ & $\begin{array}{l}\mathrm{F} \\
\mathrm{C}\end{array}$ & $\begin{array}{l}\mathrm{St} \\
\mathrm{St}\end{array}$ & $\begin{array}{c}\text { AS } \\
-\end{array}$ \\
\hline \multirow{5}{*}{ 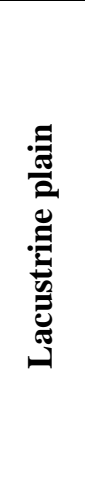 } & 25 & $\begin{array}{c}0-70 \\
70-85 \\
85-120\end{array}$ & $\begin{array}{l}10 \mathrm{YR} 7 / 3 \\
10 \mathrm{YR} 6 / 6 \\
10 \mathrm{YR} 7 / 6\end{array}$ & $\begin{array}{l}\text { SG } \\
\text { SG } \\
\text { SG }\end{array}$ & $\begin{array}{l}\text { LO } \\
\text { LO } \\
\text { LO }\end{array}$ & $\begin{array}{l}\text { LO } \\
\text { LO } \\
\text { LO }\end{array}$ & $\begin{array}{l}\text { NST } \\
\text { NST } \\
\text { NST }\end{array}$ & $\begin{array}{l}\text { NPL } \\
\text { NPL } \\
\text { NPL }\end{array}$ & $\begin{array}{l}\mathrm{C} \\
\mathrm{M} \\
\mathrm{C}\end{array}$ & $\begin{array}{l}- \\
- \\
-\end{array}$ & $\begin{array}{l}\text { St } \\
\text { St } \\
\text { St }\end{array}$ & $\begin{array}{c}\text { AS } \\
\text { AS } \\
-\end{array}$ \\
\hline & 29 & $\begin{array}{c}0-30 \\
30-80\end{array}$ & $\begin{array}{l}10 \mathrm{YR} 7 / 3 \\
10 \mathrm{YR} 7 / 3\end{array}$ & $\begin{array}{l}\text { MA } \\
\text { MA }\end{array}$ & $\begin{array}{l}\text { SO } \\
\text { SO }\end{array}$ & $\begin{array}{l}\text { FR } \\
\text { FR }\end{array}$ & $\begin{array}{l}\text { SST } \\
\text { SST }\end{array}$ & $\begin{array}{l}\text { SPL } \\
\text { SPL }\end{array}$ & $\begin{array}{l}\mathrm{C} \\
\mathrm{M}\end{array}$ & - & $\begin{array}{l}\mathrm{St} \\
\mathrm{St}\end{array}$ & $\begin{array}{c}\mathrm{AS} \\
-\end{array}$ \\
\hline & 30 & $\begin{array}{c}0-25 \\
25-80 \\
80-120\end{array}$ & $\begin{array}{l}10 \mathrm{YR} 7 / 3 \\
10 \mathrm{YR} 7 / 6 \\
10 \mathrm{YR} 7 / 3\end{array}$ & $\begin{array}{l}\text { MA } \\
\text { MA } \\
\text { MA }\end{array}$ & $\begin{array}{r}\text { SO } \\
\text { SO } \\
\text { SHA }\end{array}$ & $\begin{array}{c}\text { FR } \\
\text { FR } \\
\mathrm{Fi}\end{array}$ & $\begin{array}{c}\text { NST } \\
\text { SST } \\
\text { ST }\end{array}$ & $\begin{array}{c}\text { NPL } \\
\text { SPL } \\
\text { PL }\end{array}$ & $\begin{array}{l}\mathrm{F} \\
\mathrm{C} \\
\mathrm{M}\end{array}$ & $\begin{array}{l}- \\
\mathrm{F} \\
\mathrm{C}\end{array}$ & $\begin{array}{l}\mathrm{St} \\
\mathrm{St} \\
\mathrm{St}\end{array}$ & $\begin{array}{c}\text { AS } \\
\text { AS } \\
-\end{array}$ \\
\hline & 31 & $\begin{array}{c}0-40 \\
40-100\end{array}$ & $\begin{array}{l}\text { 10YR7/3 } \\
10 Y R 7 / 3\end{array}$ & $\begin{array}{l}\text { MA } \\
\text { MA }\end{array}$ & $\begin{array}{r}\text { SO } \\
\text { SHA }\end{array}$ & $\begin{array}{l}\mathrm{FR} \\
\mathrm{Fi}\end{array}$ & $\begin{array}{c}\text { SST } \\
\text { ST }\end{array}$ & $\begin{array}{c}\text { SPL } \\
\text { PL }\end{array}$ & $\begin{array}{l}\mathrm{C} \\
\mathrm{M}\end{array}$ & $\begin{array}{l}\mathrm{F} \\
\mathrm{C}\end{array}$ & $\begin{array}{l}\mathrm{St} \\
\mathrm{St}\end{array}$ & $\begin{array}{c}\text { AS } \\
-\end{array}$ \\
\hline & 33 & $\begin{array}{c}0-40 \\
40-110\end{array}$ & $\begin{array}{l}10 \mathrm{YR} 7 / 3 \\
10 \mathrm{YR} 7 / 3\end{array}$ & $\begin{array}{l}\text { MA } \\
\text { MA }\end{array}$ & $\begin{array}{l}\text { SO } \\
\text { SO }\end{array}$ & $\begin{array}{l}\text { FR } \\
\text { FR }\end{array}$ & $\begin{array}{l}\text { SST } \\
\text { SST }\end{array}$ & $\begin{array}{l}\text { SPL } \\
\text { SPL }\end{array}$ & $\begin{array}{l}\mathrm{C} \\
\mathrm{M}\end{array}$ & - & $\begin{array}{l}\mathrm{St} \\
\mathrm{St}\end{array}$ & $\begin{array}{c}\mathrm{CW} \\
-\end{array}$ \\
\hline
\end{tabular}


Table 2:- cont.

\begin{tabular}{|c|c|c|c|c|c|c|c|c|c|c|c|c|}
\hline \multirow{3}{*}{ 弟 } & \multirow{3}{*}{$\stackrel{0}{\stackrel{0}{0}} \stackrel{\dot{0}}{2}$} & \multirow{3}{*}{$\begin{array}{c}\text { Depth } \\
\text { (cm) }\end{array}$} & \multirow{3}{*}{$\begin{array}{c}\text { Soil } \\
\text { Color } \\
\text { (dry) }\end{array}$} & \multirow{3}{*}{ 窇 } & \multicolumn{4}{|c|}{ Consistence } & \multirow{2}{*}{\multicolumn{2}{|c|}{$\begin{array}{l}\text { Secondary } \\
\text { formation } \\
\end{array}$}} & \multirow{3}{*}{ 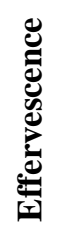 } & \multirow{3}{*}{ 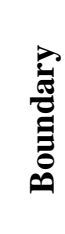 } \\
\hline & & & & & \multirow[b]{2}{*}{ Dry } & \multirow[b]{2}{*}{ Moist } & \multicolumn{2}{|c|}{ Wet } & & & & \\
\hline & & & & & & & ST & $\mathbf{P L}$ & $\mathbf{K}$ & $\mathbf{Y}$ & & \\
\hline \multirow{19}{*}{ 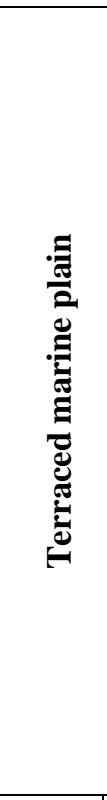 } & 1 & $0-40$ & $10 Y R 7 / 3$ & MA & $\mathrm{SO}$ & FR & SST & SPL & $\mathrm{C}$ & - & $\mathrm{St}$ & - \\
\hline & 2 & $0-40$ & 10YR7/3 & MA & $\mathrm{SO}$ & FR & SST & SPL & $\mathrm{M}$ & - & $\mathrm{St}$ & - \\
\hline & 4 & $0-25$ & $10 Y R 7 / 3$ & MA & $\mathrm{SO}$ & FR & SST & SPL & $\mathrm{M}$ & - & $\mathrm{St}$ & - \\
\hline & 5 & $0-50$ & $10 Y R 7 / 3$ & MA & $\mathrm{SO}$ & FR & SST & SPL & $\mathrm{M}$ & - & $\mathrm{St}$ & - \\
\hline & 9 & $0-30$ & $10 Y R 7 / 3$ & MA & $\mathrm{SO}$ & FR & SST & SPL & $\mathrm{M}$ & - & $\mathrm{St}$ & - \\
\hline & 12 & $0-25$ & $10 Y R 7 / 3$ & MA & $\mathrm{SO}$ & FR & SST & SPL & $\mathrm{M}$ & - & $\mathrm{St}$ & - \\
\hline & 13 & $0-25$ & $10 \mathrm{YR} 7 / 3$ & MA & $\mathrm{SO}$ & FR & NST & NPL & $\mathrm{C}$ & - & $\mathrm{St}$ & $\mathrm{CW}$ \\
\hline & 15 & $25-50$ & $10 Y R 7 / 3$ & MA & $\mathrm{SO}$ & FR & SST & SPL & M & - & $\mathrm{St}$ & - \\
\hline & & $0-25$ & $10 \mathrm{YR} 7 / 3$ & MA & $\mathrm{SO}$ & FR & NST & NPL & $\mathrm{C}$ & - & $\mathrm{St}$ & $\mathrm{CW}$ \\
\hline & 14 & $25-50$ & $10 Y R 7 / 3$ & MA & $\mathrm{SO}$ & FR & NST & NPL & M & - & $\mathrm{St}$ & - \\
\hline & 15 & $0-25$ & $10 \mathrm{YR} 7 / 3$ & MA & $\mathrm{SO}$ & FR & NST & NPL & $\mathrm{C}$ & - & $\mathrm{St}$ & - \\
\hline & 19 & $0-50$ & $10 \mathrm{YR} 7 / 3$ & MA & $\mathrm{SO}$ & FR & NST & NPL & $\mathrm{C}$ & - & $\mathrm{St}$ & - \\
\hline & 20 & $0-50$ & $10 Y R 7 / 3$ & MA & $\mathrm{SO}$ & FR & NST & NPL & $\mathrm{C}$ & - & $\mathrm{St}$ & - \\
\hline & 23 & $0-30$ & 10YR7/3 & MA & $\mathrm{SO}$ & FR & SST & SPL & $\mathrm{M}$ & - & $\mathrm{St}$ & - \\
\hline & 24 & $0-30$ & $10 \mathrm{YR} 7 / 3$ & MA & $\mathrm{SO}$ & FR & SST & SPL & $\mathrm{C}$ & - & $\mathrm{St}$ & - \\
\hline & 26 & $0-20$ & $10 \mathrm{YR} 7 / 3$ & MA & $\mathrm{SO}$ & FR & SST & SPL & $\mathrm{C}$ & - & $\mathrm{St}$ & - \\
\hline & 27 & $0-30$ & $10 \mathrm{YR} 7 / 3$ & MA & $\mathrm{SO}$ & FR & SST & SPL & $\mathrm{C}$ & - & $\mathrm{St}$ & - \\
\hline & 28 & $0-40$ & $10 \mathrm{YR} 7 / 3$ & MA & $\mathrm{SO}$ & FR & SST & SPL & $\mathrm{C}$ & - & $\mathrm{St}$ & - \\
\hline & 32 & $0-30$ & $10 \mathrm{YR} 7 / 3$ & MA & $\mathrm{SO}$ & FR & SST & SPL & $\mathrm{C}$ & - & $\mathrm{St}$ & - \\
\hline $\begin{array}{c}\text { MA } \\
\text { SG } \\
\\
\text { LO } \\
\text { SO } \\
\text { SHA } \\
\text { FR } \\
\text { HA } \\
\text { Fi } \\
\text { NST }\end{array}$ & \multicolumn{2}{|c|}{$\begin{array}{l}\text { Structure Types } \\
\text { : Massive } \\
\text { : Single grains } \\
\text { Consistence } \\
\text { : Loose } \\
\text { :Soft } \\
\text { : Slightly hard } \\
\text { :Friable } \\
\text { : Hard } \\
\text { :Firm } \\
\text { : Non Sticky }\end{array}$} & $\begin{array}{c}\text { SST } \\
\text { NPL } \\
\text { SPL } \\
\text { ST } \\
\text { PL }\end{array}$ & \multicolumn{4}{|c|}{$\begin{array}{c}\text { : Slight Sticky } \\
\text { :Non Plastic } \\
\text { :Slightly plastic } \\
\text { : Sticky } \\
\text { : Plastic } \\
\text { econdary Formation } \\
\text { Accumulation of lime } \\
\text { ccumulation of Gypsum } \\
\text { : Few } \\
\text { :common } \\
\text { :Many }\end{array}$} & $\begin{array}{c}\text { A } \\
\text { C } \\
\text { S } \\
\text { W }\end{array}$ & \multicolumn{4}{|c|}{$\begin{array}{c}\text { Effervescence } \\
\text { :Strongly effervences } \\
\text { Boundary } \\
\text { :Abrupt } \\
\text { :Clear } \\
\text { : Smooth } \\
\text { : Wavy }\end{array}$} \\
\hline
\end{tabular}


Table 3:- Some chemical and physical characteristics of the studied soil profiles.

\begin{tabular}{|c|c|c|c|c|c|c|c|c|c|c|c|}
\hline \multirow{2}{*}{$\stackrel{0}{\stackrel{0}{0}} \stackrel{\dot{0}}{\not}$} & \multirow{2}{*}{ 营 } & \multirow{2}{*}{ 플 } & \multirow{2}{*}{$\bigcup_{\underline{I}} \frac{\Xi}{\tilde{D}}$} & \multirow{2}{*}{ 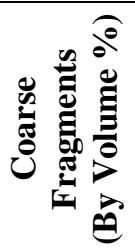 } & \multicolumn{4}{|c|}{ Grain size distribution (\%) } & \multirow{2}{*}{ 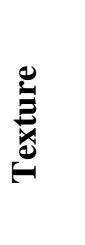 } & \multirow{2}{*}{$\bigcup_{\tilde{\sigma}}^{\infty} \delta^{\infty}$} & \multirow{2}{*}{$\sum_{b}^{\vdots}$} \\
\hline & & & & & 总 & 导 总 & 言 & $\frac{\vec{\Xi}}{\circlearrowright}$ & & & \\
\hline \multicolumn{12}{|c|}{ Gently sloping marine plain } \\
\hline \multirow[t]{2}{*}{3} & $0-25$ & 8.5 & 0.4 & 20.0 & 2.7 & 81.3 & 6.0 & 8.0 & $\mathrm{LS}$ & 33.7 & 0.67 \\
\hline & $25-70$ & 8.3 & 2.0 & 15.0 & 1.9 & 60.1 & 12.0 & 22.0 & SCL & 49.7 & 2.0 \\
\hline \multirow[t]{4}{*}{6} & $0-40$ & 8.0 & 4.0 & 0.0 & 5.2 & 66.8 & 10.0 & 18.0 & SL & 49.6 & 3.23 \\
\hline & $40-80$ & 7.0 & 15.2 & 0.0 & 7.1 & 73.9 & 7.0 & 12.0 & SL & 52.1 & 7.58 \\
\hline & $80-105$ & 7.8 & 22.4 & 0.0 & 4.2 & 73.8 & 8.0 & 14.0 & SL & 45.3 & 5.85 \\
\hline & $105-150$ & 7.8 & 15.7 & 0.0 & 3.8 & 77.2 & 7.0 & 12.0 & SL & 57.9 & 8.38 \\
\hline \multirow[t]{2}{*}{7} & $0-30$ & 8.4 & 1.4 & 0.0 & 2.9 & 79.1 & 8.0 & 10.0 & $\mathrm{LS}$ & 32.0 & 3.32 \\
\hline & $30-100$ & 7.7 & 20.0 & 0.0 & 2.1 & 63.9 & 12.0 & 22.0 & SCL & 51.2 & 10.84 \\
\hline \multirow[t]{2}{*}{8} & $0-50$ & 7.9 & 20.1 & 5.0 & 3.2 & 64.8 & 10.0 & 22.0 & SCL & 38.6 & 2.23 \\
\hline & $50-70$ & 8.1 & 3.0 & 8.0 & 1.5 & 60.5 & 14.0 & 24.0 & SCL & 42.8 & 1.55 \\
\hline \multirow[t]{3}{*}{10} & $0-30$ & 8.7 & 0.5 & 5.0 & 4.1 & 69.9 & 10.0 & 16.0 & $\mathrm{SL}$ & 41.2 & 0.67 \\
\hline & $30-80$ & 8.3 & 0.7 & 10.0 & 3.9 & 58.1 & 20.0 & 18.0 & SL & 37.8 & 0.49 \\
\hline & $80-120$ & 8.3 & 0.8 & 10.0 & 2.1 & 66.9 & 11.0 & 20.0 & SCL & 53.7 & 2.34 \\
\hline \multirow[t]{2}{*}{11} & $0-30$ & 8.6 & 0.4 & 0.0 & 3.1 & 86.9 & 4.0 & 6.0 & $\mathrm{~S}$ & 41.2 & 0.5 \\
\hline & $30-100$ & 8.6 & 0.5 & 0.0 & 3.2 & 74.8 & 8.0 & 14.0 & SL & 37.8 & 0.67 \\
\hline \multirow[t]{5}{*}{16} & $0-25$ & 8.0 & 8.2 & 2.0 & 3.1 & 54.9 & 14.0 & 28.0 & SCL & 44.5 & 0.77 \\
\hline & $25-40$ & 8.0 & 9.8 & 25.0 & 3.1 & 54.9 & 14.0 & 28.0 & SCL & 42.5 & 0.75 \\
\hline & $40-60$ & 7.5 & 11.3 & 10.0 & 2.8 & 91.2 & 2.0 & 4.0 & $S$ & 48.2 & 5.14 \\
\hline & $60-85$ & 7.8 & 16.7 & 15.0 & 1.2 & 86.8 & 4.0 & 8.0 & S & 52.1 & 5.38 \\
\hline & $85-120$ & 7.7 & 2.7 & 20.0 & 8.2 & 66.6 & 15.2 & 10.0 & SL & 62.7 & 4.84 \\
\hline \multirow[t]{3}{*}{17} & $0-30$ & 8.3 & 4.5 & 8.0 & 3.1 & 71.1 & 15.3 & 10.4 & $\mathrm{SL}$ & 47.9 & 1.04 \\
\hline & $30-70$ & 7.5 & 19.4 & 5.0 & 1.9 & 72.1 & 14.9 & 11.1 & SL & 61.3 & 4.93 \\
\hline & $70-120$ & 7.8 & 22.0 & 0.0 & 1.9 & 79.7 & 12.1 & 6.3 & LS & 69.7 & 4.84 \\
\hline \multirow[t]{2}{*}{18} & $0-25$ & 8.1 & 3.3 & 25.0 & 2.1 & 77.0 & 14.1 & 6.8 & $\mathrm{LS}$ & 17.6 & 0.67 \\
\hline & $25-70$ & 7.6 & 30.6 & 20.0 & 2.5 & 65.5 & 21.1 & 10.9 & SL & 18.5 & 0.08 \\
\hline 21 & $0-30$ & 8.2 & 5.0 & 5.0 & 1.5 & 60.7 & 26.1 & 11.7 & $\mathrm{SL}$ & 43.7 & 3.3 \\
\hline & $30-100$ & 7.9 & 19.7 & 0.0 & 1.1 & 53.2 & 30.5 & 15.2 & SL & 43.6 & 10.6 \\
\hline 22 & $0-30$ & 8.5 & 0.5 & 20.0 & 2.5 & 87.6 & 6.1 & 3.8 & $\mathrm{~S}$ & 38.8 & 3.53 \\
\hline & $30-70$ & 8.1 & 7.5 & 0.0 & 1.9 & 65.0 & 22.9 & 10.2 & SL & 50.7 & 11.41 \\
\hline & $70-120$ & 8.0 & 9.4 & 0.0 & 1.2 & 50.1 & 35.6 & 13.1 & $\mathrm{~L}$ & 45.9 & 4.84 \\
\hline 34 & $0-40$ & 8.7 & 0.5 & 0.0 & 2.1 & 73.8 & 15.1 & 9.0 & SL & 53.8 & 3.83 \\
\hline & $40-110$ & 8.4 & 2.1 & 0.0 & 1.1 & 58.2 & 29.5 & 11.2 & SL & 55.4 & 10.39 \\
\hline & & & & & trine & ain & & & & & \\
\hline & $0-70$ & 8.7 & 0.2 & 0.0 & 2.9 & 77.2 & 13.1 & 6.8 & $\mathrm{LS}$ & 45.4 & 0.32 \\
\hline 25 & $70-85$ & 8.4 & 1.4 & 0.0 & 1.9 & 84.2 & 9.1 & 4.8 & $\mathrm{LS}$ & 44.5 & 0.81 \\
\hline & $85-120$ & 8.4 & 1.8 & 0.0 & 4.3 & 79.7 & 11.1 & 4.9 & LS & 41.2 & 0.32 \\
\hline 29 & $0-30$ & 8.4 & 2.1 & 5.0 & 2.7 & 61.8 & 23.8 & 11.7 & SL & 44.7 & 0.18 \\
\hline & $30-80$ & 7.8 & 26.7 & 3.0 & 1.1 & 49.7 & 35.6 & 13.6 & $\mathrm{~L}$ & 44.9 & 0.95 \\
\hline 30 & $0-25$ & 8.9 & 0.7 & 0.0 & 1.9 & 88.1 & 5.9 & 4.1 & $S$ & 70.1 & 0.67 \\
\hline & $25-80$ & 8.1 & 9.7 & 0.0 & 1.4 & 74.4 & 15.9 & 8.3 & SL & 58.8 & 5.59 \\
\hline & $80-120$ & 8.1 & 16.6 & 0.0 & 1.6 & 65.7 & 23.1 & 9.6 & SL & 59.5 & 10.53 \\
\hline 31 & $0-40$ & 7.5 & 44.5 & 0.0 & 1.4 & 50.7 & 32.1 & 15.8 & SL & 70.2 & 2.68 \\
\hline & $40-100$ & 7.7 & 28.2 & 0.0 & 0.7 & 67.4 & 21.1 & 10.8 & SL & 39.3 & 10.79 \\
\hline 33 & $0-40$ & 8.8 & 0.6 & 0.0 & 2.6 & 71.8 & 16.9 & 8.7 & SL & 58.8 & 0.33 \\
\hline & $40-110$ & 8.5 & 4.1 & 7.0 & 1.6 & 73.0 & 16.1 & 9.3 & SL & 58.9 & 0.33 \\
\hline
\end{tabular}


Table 3:- cont.

\begin{tabular}{|c|c|c|c|c|c|c|c|c|c|c|c|}
\hline \multirow[b]{2}{*}{$\stackrel{0}{\stackrel{0}{0}} \stackrel{0}{\circ}$} & \multirow[b]{2}{*}{ 营 } & \multirow[b]{2}{*}{ 폴 } & \multirow[b]{2}{*}{$\bigcup_{x} \frac{\Xi}{\tilde{D}}$} & \multirow{2}{*}{ 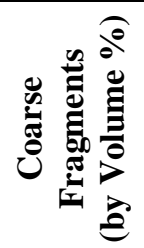 } & \multicolumn{4}{|c|}{ Grain size distribution (\%) } & \multirow[b]{2}{*}{ } & \multirow[b]{2}{*}{ 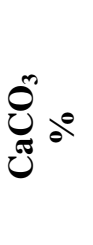 } & \multirow[b]{2}{*}{$\sum_{b}^{\Xi}$} \\
\hline & & & & & 导 & $\underset{\Xi}{\stackrel{\Xi}{\Xi}}$ & 咅 & $\frac{\vec{\sigma}}{0}$ & & & \\
\hline \multicolumn{12}{|c|}{ Terraced marine plain } \\
\hline 1 & $0-40$ & 8.2 & 1.4 & 20.0 & 2.9 & 63.1 & 13.0 & 19.0 & SL & 33.0 & 0.65 \\
\hline 2 & $0-40$ & 8.4 & 0.8 & 5.0 & 2.1 & 61.9 & 16.0 & 18.0 & SL & 16.8 & 4.37 \\
\hline 4 & $0-25$ & 8.3 & 1.5 & 20.0 & 1.2 & 80.8 & 6.0 & 10.0 & $\mathrm{LS}$ & 25.2 & 3.18 \\
\hline 5 & $0-50$ & 8.0 & 8.4 & 40.0 & 3.1 & 65.9 & 13.0 & 18.0 & SL & 47.2 & 0.25 \\
\hline 9 & $0-30$ & 8.2 & 4.9 & 5.0 & 2.2 & 63.8 & 12.0 & 22.0 & SCL & 44.5 & 1.83 \\
\hline 12 & $0-25$ & 8.4 & 0.8 & 30.0 & 2.8 & 75.2 & 8.0 & 14.0 & SL & 44.5 & 0.24 \\
\hline \multirow[t]{2}{*}{13} & $0-25$ & 8.2 & 0.8 & 0.0 & 4.1 & 63.9 & 12.0 & 20.0 & SCL & 32.8 & 0.16 \\
\hline & $25-50$ & 8.4 & 1.7 & 0.0 & 3.5 & 56.5 & 14.0 & 26.0 & SCL & 42.3 & 0.65 \\
\hline \multirow[t]{2}{*}{14} & $0-25$ & 8.4 & 0.7 & 5.0 & 2.9 & 89.1 & 4.0 & 4.0 & $\mathrm{~S}$ & 36.3 & 2.01 \\
\hline & $25-50$ & 8.4 & 0.8 & 30.0 & 2.1 & 67.9 & 12.0 & 18.0 & SL & 32.3 & 0.16 \\
\hline 15 & $0-25$ & 8.3 & 0.7 & 5.0 & 2.6 & 79.4 & 8.0 & 10.0 & LS & 36.3 & 2.69 \\
\hline 19 & $0-50$ & 8.3 & 5.7 & 7.0 & 1.7 & 57.2 & 27.2 & 13.9 & SL & 50.4 & 0.27 \\
\hline 20 & $0-50$ & 8.2 & 5.8 & 7.0 & 1.7 & 57.2 & 27.2 & 13.9 & SL & 50.4 & 0.27 \\
\hline 23 & $0-30$ & 8.5 & 1.3 & 3.0 & 1.3 & 65.5 & 22.6 & 10.6 & SL & 41.2 & 0.09 \\
\hline 24 & $0-30$ & 8.1 & 8.1 & 8.0 & 0.9 & 59.3 & 27.1 & 12.7 & SL & 37.8 & 0.33 \\
\hline 26 & $0-20$ & 8.2 & 3.4 & 8.0 & 3.2 & 60.8 & 24.9 & 11.1 & SL & 31.9 & 0.31 \\
\hline 27 & $0-30$ & 7.6 & 2.6 & 15.0 & 3.6 & 57.1 & 26.4 & 12.9 & SL & 29.4 & 1.03 \\
\hline 28 & $0-40$ & 8.1 & 4.7 & 25.0 & 2.9 & 51.8 & 30.4 & 14.9 & SL & 28.9 & 1.11 \\
\hline 32 & $0-30$ & 8.7 & 1.0 & 8.0 & 2.2 & 46.9 & 36.1 & 14.8 & $\mathrm{~L}$ & 54.1 & 0.66 \\
\hline
\end{tabular}

Land suitability for irrigated agricultural:-

The process of land suitability classification is the appraisal and grouping of specific areas of land in terms of their suitability for defined uses. The land in the survey area has been appraised to estimate its potential of suitability for irrigated agriculture. The ideal approach for land evaluation is based on evaluating the land for a specific land use. Land utilization types (LUT) are the most beneficial use of the land.

From the agricultural point of view, classification of soils for evaluating their capability for irrigation utilization aims at assessing the degree of limitation or suitability for agriculture use on the basis of their permanent properties.

\section{Current land suitability:-}

The current suitability of the studies soils was estimated by matching between the present land characteristics and their ratings according to the system outlined by Sys and Verhey (1978) and Sys et al (1991).

Suitability indices and classification of the soils profiles representing the studied geomorphic units are shown in Table (4). The results revealed that there are two suitability classes, namely:

1. Marginally suitable (S3): this class represent in all marine and lacustrine soil profiles and in about half profiles of terraced unit (Table 4).

2. Currently not suitable (N1): this class represents about the half of terraces unit profiles.

The soil limiting factors in the study area are depth (s2), texture (s1), topography (t), $\mathrm{CaCO}_{3}(\mathrm{~s} 3)$ and salinity \& alkalinity (n) with slight to severe and very severe intensity for soil limitations. 


\section{Potential Land Suitability:-}

Further land improvements are required to correct or reduce the severity of limitation exiting in the studied area, such as a) leaching of soils salinity and reclamation of soil sodicity, b) continuous application of organic manure to improve soil properties and fertility status, and c) application of drip and sprinkler irrigation system. By applying these practices, potential suitability of the studied soils could ameliorate to moderately suitable (S2) and marginally suitable (S3) (Table 4).

Table 4:- " Degree of soil limitations and suitability classes of the studied soil profiles.

\begin{tabular}{|c|c|c|c|c|c|c|c|c|c|c|c|c|c|c|c|}
\hline \multirow{3}{*}{ 离 } & \multirow{3}{*}{ 总 } & \multirow{2}{*}{\multicolumn{2}{|c|}{$\mathbf{t}$}} & \multirow[t]{3}{*}{$\mathbf{w}$} & \multicolumn{5}{|c|}{$\begin{array}{c}\text { Soil physical characteristics } \\
\text { (s) }\end{array}$} & \multirow{2}{*}{\multicolumn{2}{|c|}{$\mathrm{n}$}} & \multirow{2}{*}{\multicolumn{2}{|c|}{$\mathbf{C i}$}} & \multirow{2}{*}{\multicolumn{2}{|c|}{$\begin{array}{l}\text { Suitability } \\
\text { Class }\end{array}$}} \\
\hline & & & & & \multicolumn{2}{|c|}{ s1 } & \multirow[t]{2}{*}{ s2 } & \multirow[t]{2}{*}{$\mathbf{s 3}$} & \multirow[t]{2}{*}{ s4 } & & & & & & \\
\hline & & c & $\mathbf{p}$ & & c & $\mathbf{p}$ & & & & c & $\mathbf{p}$ & c & p & c & $\mathbf{p}$ \\
\hline \multirow{12}{*}{ 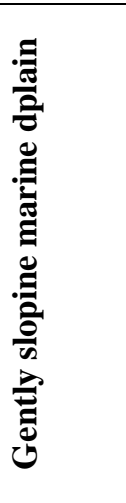 } & 3 & 75 & 100 & 100 & 65 & 75 & 75 & 90 & 100 & 90 & 100 & 29.6 & 45.6 & S3 & S3 \\
\hline & 6 & 90 & 100 & 100 & 75 & 80 & 100 & 80 & 100 & 85 & 100 & 45.9 & 64.0 & S3 & S2 \\
\hline & 7 & 90 & 100 & 100 & 80 & 85 & 90 & 90 & 100 & 85 & 100 & 49.6 & 68.8 & S3 & S2 \\
\hline & 8 & 80 & 100 & 100 & 95 & 95 & 75 & 90 & 100 & 85 & 100 & 49.1 & 64.1 & S3 & S2 \\
\hline & 10 & 75 & 100 & 100 & 80 & 85 & 100 & $\begin{array}{l}90 \\
\end{array}$ & 95 & 95 & 100 & 48.7 & 72.7 & S3 & S2 \\
\hline & 11 & 90 & 100 & 100 & 70 & 80 & 90 & 90 & 90 & 95 & 100 & 43.6 & 64.8 & S3 & S2 \\
\hline & 16 & 90 & 100 & 100 & 70 & 79 & 100 & 80 & 100 & 85 & 100 & 42.8 & 63.2 & S3 & S2 \\
\hline & 17 & 90 & 100 & 100 & 70 & 81 & 100 & 80 & 100 & 80 & 100 & 40.3 & 64.8 & S3 & S2 \\
\hline & 18 & 90 & 100 & 100 & 60 & 71 & 75 & 100 & 90 & 85 & 100 & 31.0 & 47.9 & S3 & S3 \\
\hline & 21 & 90 & 100 & 100 & 75 & 80 & 90 & 90 & 100 & 85 & 100 & 46.5 & 64.8 & S3 & S2 \\
\hline & 22 & 90 & 100 & 100 & 70 & 80 & 100 & 90 & 100 & 85 & 100 & 48.2 & 72.0 & S3 & S2 \\
\hline & 34 & 90 & 100 & 100 & 75 & 80 & 90 & 80 & 100 & 95 & 100 & 46.2 & 57.6 & S3 & S2 \\
\hline \multirow{5}{*}{ 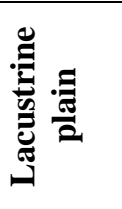 } & 25 & 75 & 100 & 100 & 55 & 65 & 100 & $\begin{array}{l}90 \\
\end{array}$ & $\begin{array}{l}90 \\
\end{array}$ & 95 & 100 & 31.7 & 52.6 & S3 & S2 \\
\hline & 29 & 90 & 100 & 100 & 80 & 85 & 90 & 90 & 90 & 85 & 100 & 44.6 & 62.0 & S3 & S2 \\
\hline & 30 & 75 & 100 & 100 & 70 & 80 & 100 & 80 & 100 & 85 & 100 & 35.7 & 64.0 & S3 & S2 \\
\hline & 31 & 75 & 100 & 100 & 75 & 84 & 90 & 90 & 100 & 75 & 100 & 34.2 & 68.0 & S3 & S2 \\
\hline & 33 & 90 & 100 & 100 & 75 & 83 & 90 & 80 & 90 & 95 & 100 & 41.6 & 53.8 & S3 & S2 \\
\hline \multirow{17}{*}{ 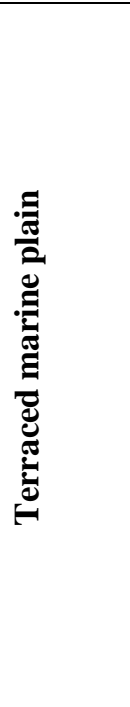 } & 1 & 75 & 100 & 100 & 65 & 75 & 55 & $\begin{array}{l}90 \\
\end{array}$ & 90 & 95 & 100 & 20.6 & 33.4 & N1 & S3 \\
\hline & 2 & 90 & 100 & 100 & 75 & 81 & 55 & 100 & 100 & 95 & 100 & 35.3 & 44.5 & S3 & S3 \\
\hline & 4 & 90 & 100 & 100 & 50 & 65 & 55 & 90 & 100 & 95 & 100 & 21.2 & 32.2 & N1 & S3 \\
\hline & 5 & 90 & 100 & 100 & 65 & 75 & 55 & 90 & 90 & 85 & 100 & 22.2 & 33.4 & N1 & S3 \\
\hline & 9 & 90 & 100 & 100 & 95 & 95 & 55 & 90 & 100 & 90 & 100 & 38.1 & 47.0 & S3 & S3 \\
\hline & 12 & 75 & 100 & 100 & 65 & 75 & 55 & 90 & 90 & 95 & 100 & 20.6 & 33.4 & N1 & S3 \\
\hline & 13 & 90 & 100 & 100 & 95 & 95 & 55 & 90 & 90 & 95 & 100 & 36.2 & 42.3 & S3 & S3 \\
\hline & 14 & 90 & 100 & 100 & 55 & 65 & 55 & 90 & 100 & 95 & 100 & 23.3 & 32.2 & N1 & S3 \\
\hline & 15 & 75 & 100 & 100 & 50 & 61 & 55 & 90 & 100 & 95 & 100 & 17.6 & 30.2 & N1 & S3 \\
\hline & 19 & 90 & 100 & 100 & 75 & 81 & 55 & 80 & 90 & 90 & 100 & 24.1 & 32.1 & N1 & S3 \\
\hline & 20 & 90 & 100 & 100 & 75 & 82 & 55 & 80 & 90 & 90 & 100 & 24.1 & 32.5 & N1 & S3 \\
\hline & 23 & 90 & 100 & 100 & 75 & 82 & 55 & 90 & 90 & 95 & 100 & 28.6 & 36.5 & S3 & S3 \\
\hline & 24 & 90 & 100 & 100 & 75 & 82 & 55 & 90 & 90 & 85 & 100 & 25.6 & 36.5 & S3 & S3 \\
\hline & 26 & 90 & 100 & 100 & 75 & 81 & 55 & 90 & 90 & 95 & 100 & 28.6 & 36.1 & S3 & S3 \\
\hline & 27 & 90 & 100 & 100 & 65 & 75 & 55 & 90 & 100 & 95 & 100 & 27.5 & 37.1 & S3 & S3 \\
\hline & 28 & 90 & 100 & 100 & 65 & 75 & 55 & 90 & 100 & 90 & 100 & 26.1 & 37.1 & S3 & S3 \\
\hline & 32 & 90 & 100 & 100 & 85 & 85 & 55 & 80 & 90 & 95 & 100 & 28.8 & 33.7 & S3 & S3 \\
\hline
\end{tabular}

Note:

$\mathrm{t}=$ Topography $\quad \mathrm{w}=$ wetness $\quad \mathrm{s} 1=$ texture $\quad \mathrm{s} 2=$ soil depth $\quad \mathrm{s} 3=\mathrm{CaCO}_{3} \quad \mathrm{~s} 4=\mathrm{CaSO}_{4} \cdot 2 \mathrm{H}_{2} \mathrm{O}$

$\mathrm{n}=$ salinity \&alkalinity $\quad \mathrm{c}=$ current $\quad \mathrm{p}=$ potential $\quad \mathrm{Ci}=$ Capability index

* no (95-100), slight (85-95), moderate (60-85) severe (45-60), very severe $(<45)$. 


\section{References:-}

1. Ali R.R. (2008) Digital Soil Mapping for Optimum Land Uses in some Newly Reclaimed Areas West of the Nile Delta, Egypt. Australian Journal of Basic and Applied Sciences, 2008, 2, 165-173.

2. Campagna M. (2005) GIS for sustainable development. Taylor \& Francis Group.

3. CONOCO (1987) Geological map of Egypt 1:50000.NH 36 NW CAIRO, Egypt.

4. EGACS (1989) Egyptian General Authority for Civil Survey. Topographic Sheets, Scales 1; 50.000.

5. El-Bastwasy, M.A. (2008) The Use of Remote Sensing and GIS for Catchments Delination in Northwestern Coast of Egypt: An Assessment of water Resources and Soil Potential., Egypt J Remote Sensing Space Sci, pp. 3-16.

6. FAO (1990) Guidelines for soil profile description, FAO, Isric. Publication, Rome, Italy

7. FAO (1976) A framework for land evaluation. FAO Soil Bulletin, 32. Rome, Italy.

8. Fehlberg, H. and Stahr, K.(1985) Development of sustained land use by understanding soil and landscape formation in the desert fringe area of nw-Egypt. CATENA, 12(1): 307-328.

9. Mineral Resources Authority of Egypt (1994) Topographic maps of Wadi El-Natroun area with 1:50,000 scales.

10. Ministry of Agriculture and Land Reclamation (2007) Climatologically normal's at Alexandria station (19712000).

11. Richards, L.A. Ed. (1954) Diagnosis and Improvement of Saline and Alkali Soils. U.S.D.A. Hand Book No. 60.

12. Said, R., 1990. The Geology of Egypt., Balkema, Rotterdam.

13. Sys, C. and Verheye, W. (1978) An attempt to the evaluation of physical land characteri-stics for irrigation according to the FAO framework for land evaluation. Int. Train. Center for post Grade. Soil Sci., Ghent, Belgium.

14. Sys, C.; Van Ramst, E.; Debaveye, J. and Beernaert, F. (1991) "Land Evaluation”. Parts I, II and III. Agric. Public. No. 7, GADC, Brussels, Belgium.

15. USDA (2004) Soil Survey Laboratory Methods Manual, Soil Survey Investigation Report No. 42, Version 4.0.

16. Zahran, A.A. (2008) Geotechnical Study of Carbonate Rocks on the Area Between Alexandria and El Alamein Along The Mediterranean Sea Coast of Egypt. Ass. Univ. Bull. Environ. Res., 11(1): 12. 